\title{
Comparison of the Effect of Low-Level Laser and Phenytoin Therapy on Skin Wound Healing in Rats
}

\author{
Jamileh Beigom Taheri' ${ }^{1}$ Fatemeh Bagheri ${ }^{1}$, Masood Mojahedi ${ }^{1}$, Nafise Shamloo' ${ }^{1}$, Mohammad Reza \\ Nakhostin', Somayyeh Azimi ${ }^{1 *}$, Zahra Namazi ${ }^{2}$
}

${ }^{1}$ School of Dentistry, Shahid Beheshti University of Medical Sciences, Tehran, Iran

${ }^{2}$ School of Dentistry, Tehran University of Medical Sciences, Tehran, Iran

\author{
Correspondence to \\ Somayyeh Azimi, DDS; School \\ of Dentistry, Shahid Beheshti \\ University of Medical Sciences, \\ Tehran, Iran \\ Tel: +98-2122403010; \\ Fax: +98-2166492992; \\ Email: \\ Somayyeh_azimi@yahoo.com
}

Published online 28 June 2015

\begin{abstract}
Introduction: Different modalities have been used to achieve better results in wound healing among which are laser and pharmacological agents. To our knowledge, there is no investigation to compare phenytoin and laser together. In this study, we aimed to evaluate laser and phenytoin efficacy and compare their effect on wound healing.

Methods: We used 60 rats. The animals were divided into 4 groups, 15 rats in each and each group was subdivided into 3 subgroups for which postoperative evaluation was performed on days 3,7 , and 14 . After general anesthesia and back hair shaving of each rat, an excisional wound was made. The wounds in group 2 were treated with $1 \%$ phenytoin cream. In group 3 , the wounds were irradiated with diode laser. The wounds of group 4 were treated with both phenytoin cream and laser administration. The animals of group 1 served as control. For histopathological evaluation, hematoxylin and eosin staining was used. Polymorphonuclear leucocytes (PMNs), re-epithelization, fibroblasts, new vessels and collagen were evaluated. Results: Faster reduction in polymorphonuclear cells after 7 and 14 days and more collagen fibers on the third day in laser treated group was statistically significant $(P<.05)$. The result for the phenytoin treated group were not better than control group. When both laser and phenytoin were used samples showed more collagen fibers on the third day, which was statistically significant $(P<.05)$.

Conclusion: It seems that low level laser is effective in skin wound healing and have better results than phenytoin application.

Keywords: LLLT; Phenytoin; Wound healing; Rat
\end{abstract}

\section{Introduction}

Restoration of structural integrity and strength of disrupted tissue of the wound depends on the interaction of healing stages. These overlapping stages include inflammation, re-epithelization, granulation tissue formation, wound contraction and tissue remodeling. Although fibroblasts and keratinocytes have a key role in wound healing, other elements such as growth factors, cytokines and their receptors as well as matrix molecules are important too. ${ }^{1}$ Wound care and the healing process are especially important to prevent complications like infection and formation of short scar tissue that compromises esthetics. ${ }^{2}$

Mester et $\mathrm{al}^{3}$ first reported the advantages of low-level laser therapy (LLLT) for wound repair. The majority of animal experiments emphasized that LLLT accelerates wound healing by enhancement of cell proliferation and formation of granulation tissue, acceleration of collagen and ATP synthesis, fostering the formation of type I and type III procollagen specific pools of mRNA, lymphocytes activation and increase in their ability to bind pathogens. ${ }^{4}$ Some other studies reported no beneficial effect for laser treatment. ${ }^{5,6}$ These contrary results may be due to different parameters like: wavelength, pulse duration, power density, pulse repetition rate, treatment repetition rate or a combination of these factors. ${ }^{7}$

The most frequently types of laser used for wound healing are helium neon $(\mathrm{HeNe})$ lasers and diode lasers, including gallium-aluminum-arsenium (GaAlAs), arsenium-gallium (AsGa), and indium-gallium-aluminum-phosphide (InGaAlP) lasers. ${ }^{8}$

There are various pharmacological agents like phenytoin that affect wound healing. ${ }^{9}$

Shapiro was the first who noticed phenytoin analgesic and healing promotion effects after gingival surgery in patients taking this medicine. After that other studies confirmed the positive effect of phenytoin in skin lesions, lichen planus, epidermolysis bullosa, peritoneal abscess, etc. ${ }^{2}$ Phenytoin promotes wound healing through different 
mechanisms. Stimulation of fibroblast proliferation, enhanced formation of granulation tissue, decreased collagenase activity, inhibition of glucocorticoid activity, direct or indirect antibacterial activity by affecting inflammatory cells, neovascularization are among them. Phenytoin also increases gene expression of the platelet derived growth factor $\beta$ chain in macrophage and monocytes. ${ }^{10}$ While most of the studies regarding phenytoin and wound healing showed phenytoin efficacy there are studies which reported no important effect. ${ }^{11-13}$

Several methods have been used to study healing promotion, but few studies investigated these modalities in comparison to each other. ${ }^{14-18}$ In this study we evaluated the effect of low-level laser in wound healing and compared it with phenytoin.

\section{Methods}

Sixty male albino rats (weighing 250-300 g) were used. The Medical Ethics Committees of Shahid Beheshti Medical University, Tehran, Iran, approved the study protocol. The animals had free access to food and water. On day zero the animals were anesthetized with peritoneal injection $(0.1 \mathrm{ml} / 100 \mathrm{~g}$ of weight $)$ of ketamine $(50 \mathrm{mg})$ and xylazine $2 \%$ (20 mg). After shaving hair of each rat on the back, the skin was disinfected with povidone iodine $10 \%$. An excisional wound was made on the back of each animal using a round biopsy punch number 5 ( 5 diameter) and the bleeding was controlled with gauze pressure on the site. The animals were randomly divided into four groups, 15 rats in each. Each group was subdivided into 3 subgroups with the same number of animals to perform the time evaluation on postoperative days 3, 7 and $14 .{ }^{19}$ The wound in group 2 was treated daily with phenytoin cream $1 \%$ for 14 days. Phenytoin was applied gently over the wounds in a fixed time. In group 3 the wounds were irradiated with a diode laser (Dr Smile-Italy) with a wavelength of $808 \mathrm{~nm}, 0.3 \mathrm{~W}$ and $0.2 \mathrm{~W}$. LLLT was started 24 hours after surgery using a restrainer to inhibit the rats from moving (Figure 1). Laser probe was situated in contact with the wound and the treatment procedure was repeated for 6 sessions ( 3 sessions per week). Every session the animals were exposed to 120 seconds of laser light with a 30 seconds rest phase between every 1 minute. The excisional wounds of group 4 were treated with both phenytoin cream and laser administration.

The animals of group 1 were neither irradiated with LLL nor phenytoin was applied for them and they served as control. Thereafter, for histopathological evaluation, 5 rats of each group were selected 3 days after surgery, 5 rat of

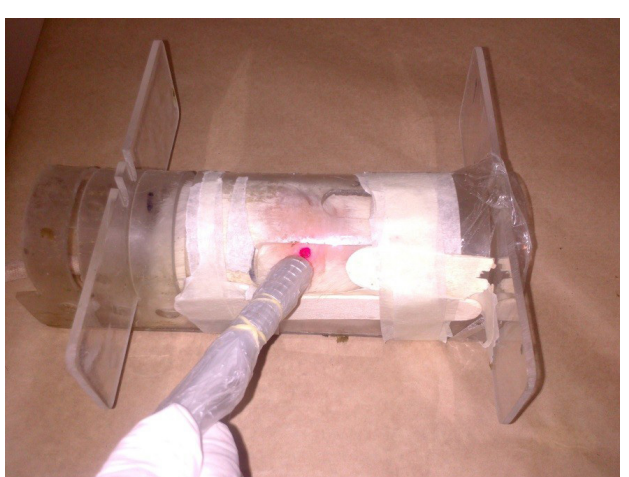

Figure 1. Using a Restrainer to Inhibit the Rats From Moving.

each group were selected 7 days after surgery and 5 rats 14 days after surgery and the samples were obtained using a biopsy punch number 8 (with 8 diameters). Then the specimens were stained with hematoxylin and eosin and were examined by microscope. A semi-quantitative method was used to evaluate the following histological features: polymorphonuclear leucocytes (PMNs), re-epithelization, fibroblasts, new vessels and collagen. The sections were evaluated in a blinded manner on a scale of $0-4$ (Table 1 ). ${ }^{20}$

Statistical Analysis

Data were analyzed by chi-square and Kruskal-Wallis tests with SPSS version 19.

\section{Results}

Results are described below in 3 parts according to the days after wound creation.

\section{Three Days After Surgery}

In group $\mathrm{A}$ the histopathological reading after 3 days showed keratinocytes migration beneath the wound, which covered the gap. Granulation tissue (GT) formation together with moderate fibroblast proliferation and infiltration of PMNs was seen. In addition a lot of congested blood vessels and a few collagen fibers were present in GT. Re-epithelization, marked infiltration of PMNs, fibroblast proliferation were also seen in the samples of group C and $\mathrm{D}$ which were treated with laser and more newer collagen fibers were present in these 2 groups compared to control group, which was statistically significant $(P<.05)$. (Figures 2A-C; Figure 3)

Seven Days After Surgery

At 7 days postsurgery, epithelization was complete in all

\begin{tabular}{lcccc}
\hline Scale & Epithelization & PMNs & Fibroblast & New Vessels \\
\hline 0 & Thickness of cut edges & Absent & Absent & Absent \\
1 & Migration of cells (<50\%) & Mild ST & Mild ST & Mild SCT \\
2 & Migration of cells (>50\%) & Mild GT & Mild GT & Mild GT \\
3 & Bridging the excision & Moderate GT & Moderate GT & Moderate GT \\
4 & Keratinization & Marked GT & Marked GT & Marked GT \\
\hline
\end{tabular}

Abbreviations: ST, surrounding tissue; GT, granulation tissue, SCT, subcutaneous tissue. 

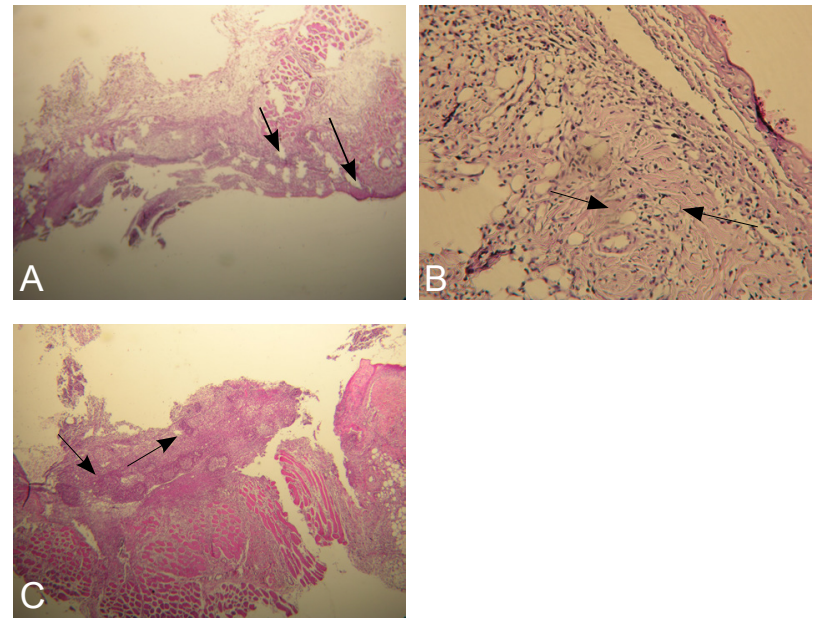

Figure 2. Three Days Postsurgery: Healing with granulation tissue (GT) formation composed of new vessels and dense infiltration of lymphocytes and plasma cells is seen. More collagen fibers are seen in laser group sample. (A) Control group: Granulation Tissue (GT) formation composed of new vessels [long arrow] and dense infiltration of lymphocytes and plasma cells (short arrow). (B) Laser group: GT formation composed of new vessels and dense infiltration of lymphocytes and plasma cells. More collagen fibers are seen in laser group sample (black arrows). (C) Phenytoin group: GT formation composed of new vessels (short arrow) and dense infiltration of lymphocytes and plasma cells (long arrow).

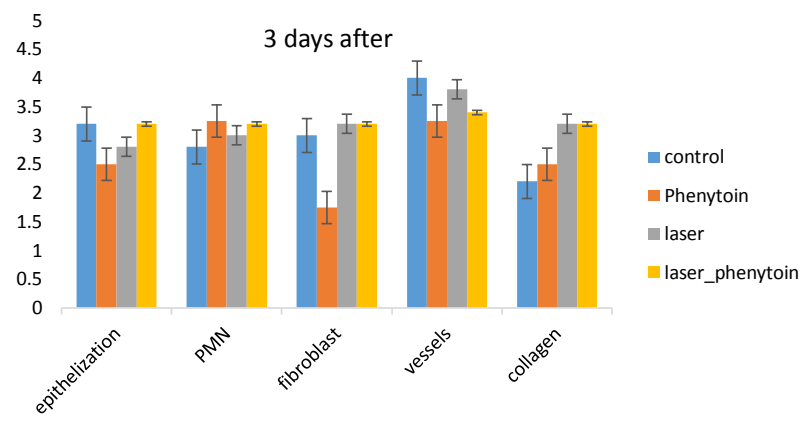

Figure 3. Semi-quantitative Analysis of Histological Structures/ Changes: 3 Days After Surgery.

samples of 4 groups and keratin layer was produced although in phenytoin group the healing process was slower. Fibroblasts were markedly present in GT as well as few new vessels in surrounding connective tissue and dense collagen fibers within GT. All samples showed marked reduction in PMNs (stage 1) but it was more obvious in group $\mathrm{C}$ which was treated with laser. There was the least PMNs in samples of group $C$ while other samples yet had been infiltrated with PMNs in surrounding tissue and it was statistically significant $(P>.05)$. (Figures $4 \mathrm{~A}, \mathrm{~B}$; Figure 5).

\section{Fourteen Days After Surgery}

After 14 days, there were fewer differences between groups and the samples of phenytoin treated group showed complete re-epithelization and dense collagen fibers in sur-

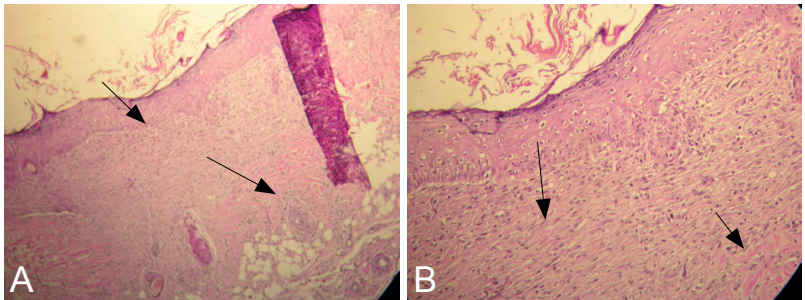

Figure 4. Seven Days Postsurgery: Healing with complete formation of orthokeratinized epithelium and the presence of fibroblasts and collagen fibers with few lymphocytes infiltration. (A) Control group: 7 days postsurgery: Healing with complete formation of orthokeratinized stratified squamous epithelium (short arrow) and the presence of fibroblasts and collagen fibers (long arrow) with few lymphocytes infiltration. (B) Laser group: 7 days postsurgery: Healing with complete formation of orthokeratinized stratified squamous epithelium and the presence of fibroblasts (long arrows) and collagen fibers (short arrow) with few lymphocytes infiltration.

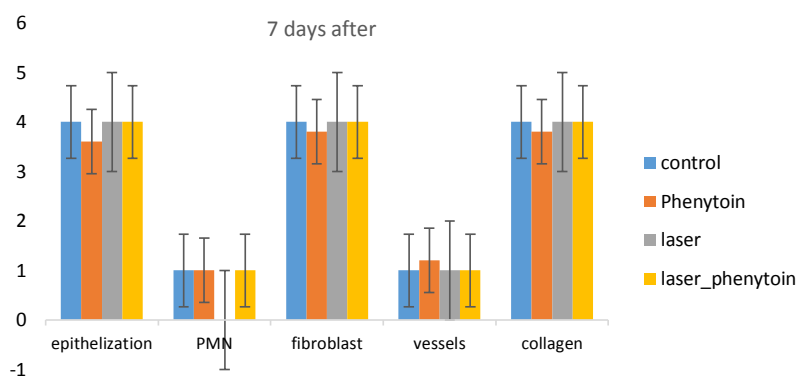

Figure 5. Semi-quantitative Analyses of Histological Structures/ Changes: 7 Days After Surgery.

rounding tissue. For other groups the results were almost the same as 7 days after surgery and inflammation was yet at the minimum level for laser treated group (Figure 6 and Figure 7).

\section{Discussion}

To assess wound regeneration, the following histological criteria were evaluated: extent of re-epithelization, maturation and organization of the epidermal cells, angiogenesis, fibroblast proliferation and intensity of inflammation. ${ }^{1}$ LLLT with diode $808 \mathrm{~nm}$ resulted in more collagen in day 3 and less PMNs after 7 and 14 days compared to nonlaser groups. Phenytoin application did not resulted in better wound healing, there were even less fibroblasts and slower re-epithelization in this group. The combination of phenytoin and laser had better result in contrast to phenytoin alone and more collagen fibers were present. Moreover we have also found that LLLT is able to stimulate hair growth. Our result is in agreement with studies in which increase in collagen fibers and faster elimination of inflammation was reported after laser administration.

Lacjaková et $\mathrm{al}^{21}$ reported reduced inflammation and faster re-epithelization following laser administration at different doses. Dawood and Salman showed faster re-epithelization and more collagen fibers and revealed 


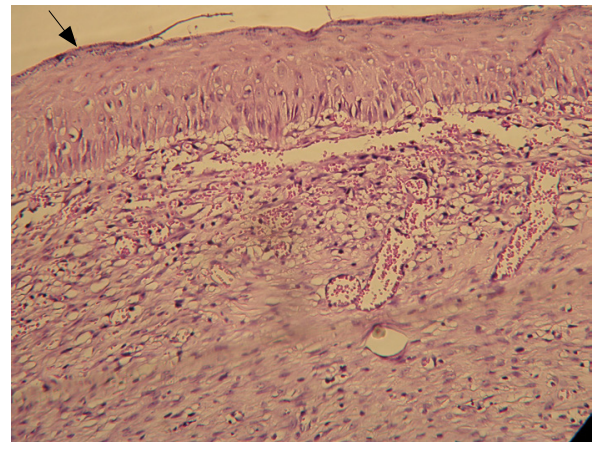

Figure 6. Fourteen Days Postsurgery: Healing with complete formation of orthokeratinized stratified squamous epithelium and the presence of fibroblasts and collagen fibers with few lymphocytes infiltration.

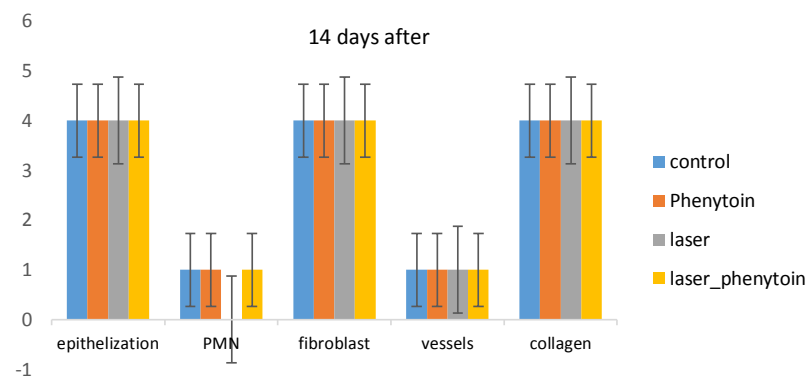

Figure 7. Semi-quantitative Analyses of Histological Structures/ Changes: 14 Days After Surgery.

that low-level diode laser accelerates wound healing, ${ }^{22}$ although the type of wound model was different in these studies. Busnardo and Biondo-Simões ${ }^{19}$ reported increase in collagen in day 3 postsurgery and less PMNs after laser treatment which is in agreement with our study.

Laser administration resulted in less inflammation and more collagen fibers in our study but not faster re-epithelization. In Viegas et $\mathrm{al}^{23}$ study laser energy did not minimize tissue inflammatory reactions although the evaluation method used was PCR. Some other authors failed to show wound healing acceleration after laser irradiation although these conflicting results could be attributed to the varying irradiance, exposure time and incorrect control groups. ${ }^{5,624} \mathrm{~A}$ variety of reasons are mentioned for such differences in investigation results. Different parameters should be considered in laser treatment such as wavelength, pulse duration, power density, pulse repetition rate and treatment repetition rate. Lack of accurate documentation of irradiation protocol and appropriate control group as well as poor methodological quality are the weak points of studies in which laser effect on wound healing is assessed. Different wound models and wound with different etiology as well as systemic effect of laser when each animal is its own control can mask the results. ${ }^{7,25,26}$

Chemical drugs, such as phenytoin are available for wound healing too. Despite the large amount of studies, which confirm the efficacy of phenytoin for wound heal- ing, in our study, phenytoin group was not better than the control group. Muthukumarasamy et al, ${ }^{27}$ Hasamnis et al, ${ }^{28}$ and Oluwatosin et a ${ }^{17}$ all reported faster healing and more reduction in wound area following the use of phenytoin. Although some authors did not achieve statistically significant results when using this drug. ${ }^{12,13}$ Regardless of the different etiology and nature of the wounds in different studies, which affect the results, it is believed that phenytoin results in a hypertrophic GT and increased collagen synthesize. ${ }^{29,30}$ According to this, it seems that a small shallow wound in a rat that close within a few days or an incisional wound, which heals with a minimal amount of GT, are not good models to reflect the effect of phenytoin. In addition the distinct situations in human studies like involvement with different systemic diseases and different drugs, which inhibit healing, the extend of the wound and different evaluation methods are other factors that influence the results.

\section{Conclusion}

In this study laser administration had a positive effect on wound healing, but phenytoin application did not lead to better results.

\section{Conflict of Interest}

The authors have no conflict of interest to declare.

\section{Acknowledgments}

This article was based on a postgraduate thesis by Dr. Bagheri, which was successfully completed under the supervision of Dr. Taheri with the close cooperation of the Oral Medicine Department of Dental School, Shahid Beheshti University of Medical Sciences.

\section{References}

1. Thakur R, Jain N, Pathak R, Sandhu SS. Practices in wound healing studies of plants. Evid Based Complement Alternat Med. 2011;2011:438056. doi:10.1155/2011/438056.

2. Kadkhodazadeh M, Khodadoustan A, Seif N, Amid R. Short-term effects of $1 \%$ topical phenytoin suspension on the donor site. Pain and wound size after free gingival grafts. J Dent School. 2012;29(5):366-372.

3. Mester E, Nagylucskay S, Döklen A, Tisza S. Laser stimulation of wound healing. Acta Chir Acad Sci Hung. 1976;17(1):49-55.

4. Woodruff LD, Bounkeo JM, Brannon WM, et al. The efficacy of laser therapy in wound repair: a metaanalysis of the literature. Photomed Laser Surg. 2004; 22:241-247. doi:10.1089/1549541041438623.

5. Anneroth G, Hall G, Rydén H, Zetterqvist L. The effect of low-energy infra-red laser radiation on wound healing in rats. Br J Oral Maxillofac Surg. 1988;26(1):12-17.

6. Colver GB, Priestley GC. Failure of a helium-neon laser to affect components of wound healing in vitro. Br J Dermatol. 1989;121(2):179-186.

7. Lucas C, Stanborough RW, Freeman CL, De Haan RJ. 
Efficacy of low-level laser therapy on wound healing in human subjects: a systematic review. Lasers Med Sci. 2000;15:84-93. doi:10.1016/0266-4356(88)90144-1

8. da Silva JP, da Silva MA, Almeida AP, Lombardi Junior I, Matos AP. Laser therapy in the tissue repair process: a literature review. Photomed Laser Surg. 2009; 27(6):929-935.

9. Swamya SM, Tanb P, Zhu YZ, Lu J, Achuth HN, Moochhala S. Role of phenytoin in wound healing: microarray analysis of early transcriptional responses in human dermal fibroblasts. Biochem Biophys Res Commun. 2004;314:661-666. doi:10.1016/j. bbrc.2003.12.146.

10. Tauro LF, Shetty P, Dsouza NT, Mohammed S, Sucharitha S. A comparative study of efficacy of topical phenytoin vs conventional wound care in diabetic ulcers . Int J Mol Med Sci. 2013;3:65-71.

11. Subbanna PK, Margaret FX, George J, et al. Topical phenytoin solution for treating pressure ulcers: a prospective, randomized, double-blind clinical trial. Spinal Cord. 2007; 45(11): 739-743.

12. Mirnezami M, Ebrahimi Fakhar HR, Rezaei K, Rahimi H. Comparing the healing effects of topical phenytoin, conjugated estrogen and silver sulfadiazine on skin wounds in male rats. J Kashan Univ Med Sci. 2011;15:11-16.

13. Tabatabaei Naeini A, Oryan A, Dehghani S, Nikahval B. Effects of topical and parenteral application of phenytoin on cutaneous wound healing in rabbits. J Anim Vet Adv. 2008;7:1537-1545. doi:10.1007/ s00580-008-0724-4.

14. Salman H, Kashmoola MA, Al-Waiz MM, AlSandooq TA. Differences between low level laser therapy and triamcinolone acetonide kenalog on healing of recurrent aphthous ulceration. Ann Coll Med Mosul. 2008;34(1):35-41.

15. Demir H, Yaray S, Kirnap M, Yaray K. Comparison of the effects of laser and ultrasound treatments on experimental wound healing in rats. J Rehab Res Dev. 2004;41(5):721-728. doi:10.1682/jrrd.2003.08.0131.

16. Hollisaz MT, Khedmat H, Yari F. A randomized clinical trial comparing hydrocolloid, phenytoin and simple dressings for the treatment of pressure ulcers. BMC Dermatol. 2004;4:18. doi:10.1186/14715945-4-18.

17. Oluwatosin OM, Olabanji JK, Oluwatosin OA, Tijani LA, Onyechi HU. A comparison of topical honey and phenytoin in the treatment of chronic leg ulcers. Afr J Med Med Sci. 2000;29(1):31-34.

18. Santuzzi CH, Buss HF, Pedrosa DF, Freire MO, Nogueira BV, Gonçalves WL. Combined use of low level laser therapy and cyclooxygenase-2 selective inhibition on skin incisional wound reepithelialization in mice: a preclinical study. An Bras Dermatol. 2011;86(2):278-283.
19. Busnardo VL, Biondo-Simões ML. Effects of lowlevel helium-neon laser on induced wound healing in rats. Rev Bras Fisioter. 2010;14:45-51.

20. Gal P, Kilik R, Mokry M, et al. Simple method of open skin wound healing model in corticosteroid-treated and diabetic rats:standardization of semi-quantitative and quantitative histological assessments. Vet Med. 2008;53:652-659.

21. Lacjaková K, Bobrov N, Poláková M, et al. Effects of equal daily doses delivered by different power densities of low-level laser therapy at $670 \mathrm{~nm}$ on open skin wound healing in normal and corticosteroidtreated rats: a brief report. Lasers Med Sci. 2010; 41(7):1038-1042. doi:10.1007/s10103-010-0791-z.

22. Dawood MS, Salman SD. Low level diode laser accelerates wound healing. Lasers Med Sci. 2013;28: 941-5.

23. Viegas VN, Abreu ME, Viezzer C, Machado DC, Filho MS, Silva DN, Pagnoncelli RM.Effect of low-level laser therapy on inflammatory reactions during wound healing: comparison with meloxicam. Photomed Laser Surg. 2007;25(6):467-473. doi:10.1089/pho.2007.1098.

24. Allendorf JD, Bessler M, Huang J, et al. Heliumneon laser irradiation at fluences of 1,2 , and $4 \mathrm{~J} / \mathrm{cm} 2$ failed to accelerate wound healing as assessed by both wound contracture rate and tensile strength. Lasers Surg Med. 1997;20:340-345.

25. Lucas C, Criens-Poublon LJ, Cockrell CT, de Haan RJ. Wound healing in cell studies and animal model experiments by low level laser therapy; were clinical studies justified? a systematic review. Lasers Med Sci. 2002;17:110-134. doi:10.1007/s101030200018.

26. Pirilä E, Parikka M, Ramamurthy NS, et al. Chemically modified tetracycline (CMT-8) and estrogen promote wound healing in ovariectomized rats: effects on matrix metalloproteinase-2, membrane type 1 matrix metalloproteinase, and laminin-5 gamma2-chain. Wound Repair Regen. 2002;10:38-51. doi:10.1046/ j.1524-475x.2002.10605.x.

27. Muthukumarasamy MG, Sivakumar G, Manoharan G. Topical phenytoin in diabetic foot ulcers. Diabetes Care. 1991;14(10):909-911. doi:10.2337/ diacare.14.10.909.

28. Hasamnis A, Mohanty B, Muralikrishna, Patil S. Evaluation of wound healing effect of topical phenytoin on excisional wound in albino rats. J Young Pharm. 2010;2(1):59-62.

29. Deshmukh VS, Motghare VM, Padwal SL, et al. Effect of phenytoin in wound healing in albino rats. Int $J$ Exp Pharmacol. 2014;4(1):4-7.

30. Nidal Y, Abla A, Darwish B, Salwan O. Wound bed preparation with 10 percent phenytoin ointment increases the take of split - thickness skin graft in large diabetic ulcers. Dermatol Online J. 2006;12(6): 5. 